\title{
The respiratory and metabolic responses for determinants of exercise endurance time in constant-load exercise test in healthy adult volunteers, prospective cross-sectional design
}

Shohei Kawachi

Shinshu Daigaku https://orcid.org/0000-0002-5093-5125

Keisaku Fujimoto ( $\square$ keisaku@shinshu-u.ac.jp )

https://orcid.org/0000-0002-6512-1757

Research article

Keywords: cardiopulmonary exercise test, anaerobic threshold, ventilatory inefficiency, exercise tolerance

Posted Date: May 10th, 2020

DOI: https://doi.org/10.21203/rs.3.rs-26699/v1

License: (c) (1) This work is licensed under a Creative Commons Attribution 4.0 International License.

Read Full License 


\section{Abstract}

Although constant-load exercise test is performed at approximately $80 \%$ of maximum load determined based on individual exercise capacity of incremental-load exercise test, some subjects cannot maintain exercising in constant-load exercise test. We assumed that the metabolic responses difference between those who are and are not able to maintain exercising in only constant-load exercise. Therefore, present study was performed to elucidate the metabolic responses for determinants of endurance time in constant-load exercise test. Twenty-seven healthy volunteers performed constant-load exercise test at $80 \%$ of maximum load determined by incremental-load exercise test. Subjects were separated into those who were and were not able to complete 20 minutes of constant-load exercise test. The metabolic indexes were compared between the two groups. The not-completed group showed significantly higher respiratory exchange ratio (RER) as anaerobic threshold and minimum minute ventilation/carbon dioxide production $\left(\mathrm{VE} / \mathrm{V}_{\mathrm{CO} 2}\right)$ as ventilatory inefficiency at peak exercise only in constant-load exercise test $(P<$ 0.05 ). It was suggested that the respiratory and metabolic responses for determinants of endurance time in constant-load exercise test were exercise beyond anaerobic threshold and the increase in $\mathrm{CO}_{2}$ output due to ventilatory inefficiency related to a reduced skeletal muscle in healthy adults.

\section{Introduction}

Cardiopulmonary exercise test (CPET) is performed to evaluate cardiovascular, respiratory, and metabolic function in patients or healthy subjects in clinical practice and in sports science. ${ }^{1-2}$ CPET consists mainly of incremental-load exercise test (ILET) and constant-load exercise test (CLET). Generally, ILET evaluates maximum oxygen intake as exercise tolerance and respiratory metabolism by exercising until limited by symptoms with gradually increasing exercise load and used for evaluation of treatment efficacy. ${ }^{1}$ CLET has been used to evaluate kinetic oxygen intake and dynamic lung hyperinflation in chronic obstructive pulmonary disease (COPD) and is performed at approximately $70 \%-80 \%$ of peak $\mathrm{Vo}_{2}$ exercise capacity determined in ILET., ${ }^{1,4}$ As CLET has been reported to reflect the treatment efficacy of pharmacological and non-pharmacological treatments compared with ILET, CLET has been used for evaluation of the effects of bronchodilators, oxygen therapy, and pulmonary rehabilitation. ${ }^{5-7}$ As the metabolic kinetics reaches a plateau at $5-10$ minutes after commencement of exercise in CLET, it is recommended that the subject maintain exercising for more than $5-10$ minutes. ${ }^{8}$ Exercise endurance time of CLET is adopted as exercise tolerance and is often the upper limit up to $20-30$ minutes. $^{9-10}$ Subjects are separated into those who are and are not able to maintain exercising for a longer duration in CLET even though CLET is performed with a load based on individual exercise capacity determined by ILET. ${ }^{10}$ The reason why some healthy subjects cannot maintain exercising in only CLET has not been elucidated. A previous study reported that CLET more reflect metabolism of muscle and had a larger metabolic responses such as progresses of lactic acidosis compared to ILET. ${ }^{11}$ Therefore, we assumed that the respiratory and metabolic responses which are different between those who are and are not able to maintain exercising in CLET affects exercise endurance of CLET even though CLET is performed with a load based on individual exercise capacity determined by ILET. The present study was performed to 
elucidate the respiratory and metabolic responses for determinants of maximum exercise endurance time during CLET in healthy adult volunteers.

\section{Materials And Methods}

\section{Subjects}

The study population consisted of 27 healthy adult volunteers ( 24 male and 3 female) from 18 to 70 years old examined between March 2017 and December 2018.

\section{Study design}

This study had a prospective cross-sectional design. All subjects underwent spirometry. Symptom-limited ILET was performed, and then CLET was conducted at a load of $80 \%$ of the maximum exercise capacity determined by ILET. The endurance time of CLET was upper limit to 20 minutes. The subjects were divided into those who were and were not able to complete 20 minutes of exercise in CLET. We designated these groups as the completed group and the not-completed group, respectively, and compared metabolic indexes in each exercise test between the groups to elucidate the respiratory and metabolic responses for determinants of exercise endurance time in CLET.

\section{Spirometry}

Spirometry was performed using a spirometer (Fukuda Denshi Co., Ltd., Tokyo, Japan). All measurements were performed according to the Japanese Respiratory Society guidelines for lung function measurements. For predicted values of $\mathrm{FEV}_{1}$ and $\mathrm{VC}$, Japanese local reference data developed by the Japanese Respiratory Society were adopted. ${ }^{12}$

\section{Symptom-limited incremental-load exercise test}

Both exercise tests were performed using a treadmill (Auto Runner AT-200; Minato Medical Science Co., Ltd., Osaka, Japan), and methods were referenced to previous studies. ${ }^{10}$ Oxygen uptake $\left(\mathrm{V}_{\mathrm{O} 2}, \mathrm{ml} / \mathrm{kg} / \mathrm{min}\right)$; carbon dioxide production $\left(\mathrm{V}_{\mathrm{CO} 2}, \mathrm{ml} / \mathrm{kg} / \mathrm{min}\right)$; Respiratory exchange ratio $\left(\mathrm{RER}=\mathrm{V}_{\mathrm{CO} 2} / \mathrm{V}_{\mathrm{O} 2}\right)$; end-tidal oxygen ( $\left.\mathrm{ET}_{\mathrm{O} 2}, \%\right)$; end-tidal carbon dioxide $\left(\mathrm{ET}_{\mathrm{CO}}, \%\right)$; minute ventilation/oxygen uptake $\left(\mathrm{VE} / \mathrm{V}_{\mathrm{O} 2}\right)$; minute ventilation/carbon dioxide production $\left(\mathrm{VE} / \mathrm{V}_{\mathrm{CO}}\right)$; minute ventilation $(\mathrm{VE}, \mathrm{L} / \mathrm{min})$; tidal volume $(\mathrm{VT}, \mathrm{L})$; and respiratory rate (RR, times/min) were recorded using the breath-by-breath measurements in each exercise test determined using a breath analyzer system (AE-310S Aeromonitor; Minato Medical Science). These metabolic indexes during exercise measured in a breath-by-breath manner were assessed by the average value for $10 \mathrm{~s}$ before the end of exercise, which was called the "peak value." We evaluated the anaerobic threshold (AT) point by the V-Slope method and respiratory compensation (RC) point was determined by the VE- $\mathrm{V}_{\mathrm{CO} 2}$ slope method according to ATS/ACCP statement respectively. ${ }^{1}$ Percutaneous oxygen saturation $\left(\mathrm{SP}_{\mathrm{O} 2}, \%\right)$ and pulse rate $(\mathrm{PR}$, beats/min) during exercise were measured continuously with a fingertip monitor (AE-310S Aeromonitor; Minato Medical Science). 
The protocol of ILET with a treadmill adopted TR-3 which is designed to increase $\mathrm{V}_{02}$ linearly. In this protocol, the speed and slope changed continuously and was the same method as used in determination of the AT reference value in Japanese subjects. ${ }^{13}$ Subjects continued to exercise until limited by symptoms and maximum exercise capacity and maximum exercise load were evaluated. Subjects walked on a treadmill at a speed of $1 \mathrm{~km} / \mathrm{h}$ with a slope of $0 \%$ for 3 minutes before and after ILET as warm-up and cool-down.

\section{Symptom-limited constant-load exercise test}

After sufficient rest, symptom-limited CLET was conducted at a load of $80 \%$ of the maximum load in the ILET as in the previous study. ${ }^{10}$ First, the subjects walked for 3 minutes as warm-up at a speed of $1 \mathrm{~km} / \mathrm{h}$ with a slope of $0 \%$ on the treadmill. Next, the subject began exercise at up to $80 \%$ of the maximum load of ILET. The CLET was performed to the point when the subject could no longer maintain exercise or a time limit of 20 minutes had been reached. ${ }^{10}$ After CLET, the subjects walked for 3 minutes as a cooldown with the same load as in the warm-up. The subjects who were not able to complete 20 minutes of exercise in CLET were evaluated whether they did maximum-effort exercise in consideration of $85 \%$ of maximal age-predicted heart rate. ${ }^{14}$ The subjects were divided into those who were and were not able to complete 20 minutes of exercise in CLET, defined as the completed group and the non-completed group, respectively.

\section{Statistical analysis}

As there have been no similar previous studies, sample size could not be calculated. Therefore, we calculated the statistical power by post hoc analysis. Normality was analyzed by the method of Kolmogorov-Smirnov and then Student's $t$ test was used for comparison of metabolic indexes between the completed group and the non-completed group. Finally, single logistic regression analysis was conducted with the completed or non-completed group as the independent variable and metabolic indexes that were significantly different between the two groups as the explanatory variable. In all analyses, $P<0.05$ was taken to indicate statistical significance. SPSS ver. 25 was used for statistical analyses (SPSS Inc., Chicago, IL).

\section{Results}

\section{Characteristics of physical findings, lung function, and complications}

Table 1 shows the characteristics of physical findings and spirometry. A total of 27 subjects were enrolled in this study. Physical findings and lung functions were not significantly different between the two groups and all values of spirometry were within reference ranges in all subjects.

\section{Comparison of metabolic indexes in incremental-load exercise test}


Table 2 shows the results of comparison in ILET. The peak $\mathrm{V}_{\mathrm{O} 2}$ as a general index of exercise tolerance were $43.4 \pm 2.1$ and $47.5 \pm 3.0$ in the completed group and the non-completed group, respectively, which were within the reference range and were not significantly different. The AT point and RC point were not different between the two groups and were within the reference ranges in Japanese subjects. The other metabolic indexes were also not significantly different between the two groups.

\section{Comparison of metabolic indexes in constant-load exercise test}

Table 3 shows the results of CLET. The not-completed group showed significantly higher values of peak

$\mathrm{V}_{\mathrm{CO} 2}, \mathrm{RER}$ and $\mathrm{VE}$ in comparison to the completed group. The RER in the not-completed group exceeded 1 (Fig. 1). The minimum VE/ $\mathrm{V}_{\mathrm{CO} 2}$, as an index of ventilatory inefficiency, was significantly higher in the notcompleted group than the completed group, whereas $\mathrm{VE} / \mathrm{V}_{\mathrm{CO} 2}$ at peak in CLET were not different between the two groups. The peak $V_{02}$ of non-completed group increased until near the RC point of ILET. However, $\mathrm{VE} / \mathrm{V}_{\mathrm{CO} 2}$ which increase after exceeding RC point did not show a significant difference between the two groups.

\section{Results of logistic regression analysis and comparison between the two exercise tests}

Table 4 shows the results of single logistic regression analysis with regard to the metabolic indexes. The minimum VE/ $/ \mathrm{V}_{\mathrm{CO} 2}$ showed the most significant $P$-value and $95 \%$ confidence interval in the regression model to predict the completion of exercise $(P=0.05)$, and the subjects with lower values of minimum $\mathrm{VE} / \mathrm{V}_{\mathrm{CO} 2}$ could complete the 20 minutes of exercise.

\section{Discussion}

This study was performed to elucidate the respiratory and metabolic responses for determinants of exercise endurance time in CLET in healthy volunteers. The study population consisted of 27 healthy adult volunteers. CLET was conducted at a load of $80 \%$ of the maximum exercise capacity. The subjects were divided into those who were and were not able to complete 20 minutes of exercise (completed group and not-completed group, respectively). We compared metabolic indexes between the two groups. The not-completed group showed significantly higher RER and peak VE than the completed group in CLET, and the RER exceeded 1 as the standard of AT. The minimum VE/ $\mathrm{V}_{\mathrm{CO} 2}$ as an index of ventilatory inefficiency was significantly higher in the not-completed group. Single logistic regression analysis showed that the minimum VE/ $\mathrm{V}_{\mathrm{CO} 2}$ had the greatest predictive capability in the regression model for completing the exercise. It was suggested that the respiratory and metabolic responses for determinants of exercise endurance time in CLET was exercise beyond AT due to ventilatory inefficiency related to a reduced skeletal muscle in healthy adults. 
$\operatorname{RER}\left(\mathrm{V}_{\mathrm{CO} 2} / \mathrm{V}_{\mathrm{O} 2}\right)$ is used as an index of AT when RER exceeds $1,{ }^{15}$ and $A T$ is correlated with exercise endurance time. ${ }^{16}$ In the present study, the RER was significantly higher and exceeded 1 in the notcompleted group. Therefore, the endurance time in the not-completed group could have been restricted by anaerobic metabolism in comparison with the completed group even though the subjects performed CLET based on their individual exercise capacity of ILET. Ventilation is more enhanced to compensate for the increased $\mathrm{CO}_{2}$ production exceeding AT. ${ }^{17}$ Therefore, the not-completed group showed higher $\mathrm{V}_{\mathrm{CO} 2}$ and VE at peak in CLET. Since the peak $\mathrm{V}_{02}$ of the not-completed group increased until near the RC point of ILET, the non-completed group may have exercised to near the RC point in CLET. However, as VE/ $\mathrm{V}_{\mathrm{CO} 2}$ which increase after RC point did not show a significant difference between the two groups, the notcompleted group may not have exceeded the RC point in CLET.

The not-completed group showed not only a higher RER but also a higher minimum VE/ $\mathrm{V}_{\mathrm{CO} 2}$ as an indicator of ventilatory inefficiency. This could have been caused by enhanced $V_{\mathrm{CO} 2}$ production due to reduced skeletal muscle or metabolic inefficiency of the muscles. Although minimum VE/ $\mathrm{V}_{\mathrm{CO} 2}$ as an indicator of ventilatory inefficiency generally reflects V/Q mismatch in patients with heart failure, ${ }^{18}$ in healthy subjects, it is related to the mass and strength of skeletal muscle. ${ }^{19}$ Gonzales et al. also reported a correlation between VE/ $\mathrm{V}_{\mathrm{CO} 2}$ at $\mathrm{AT}$ and the lower limb muscle strength in healthy adults. Furthermore, Keller-Ross et al. reported that minimum VE/ $\mathrm{V}_{\mathrm{CO} 2}$ and RER affected by increasing $\mathrm{V}_{\mathrm{CO} 2}$ were related to the type of fibers in skeletal muscle. ${ }^{20}$ Therefore, it was suggested that the increased RER and minimum $\mathrm{VE} / \mathrm{V}_{\mathrm{CO} 2}$ in the not-completed group may have been caused by reduced skeletal muscle, which resulted in decreased exercise endurance time in CLET, and that the minimum VE/ $\mathrm{V}_{\mathrm{CO} 2}$ was the most accurate variable to predict exercise endurance time in logistic regression analysis.

The reason why there were differences in AT and ventilatory inefficiency related to a reduced skeletal muscle in CLET despite the lack of significant differences at all metabolic indexes in ILET is that there were differences in metabolic responses between ILET and CLET. Yamamoto et al. reported that lactic acidosis is more caused by a larger $\mathrm{O}_{2}$ extraction in CLET compared to ILET. ${ }^{11} \mathrm{O}_{2}$ extraction is the release of $\mathrm{O}_{2}$ from hemoglobin, and this phenomenon is facilitated by the Bohr effect when subjects exceed $\mathrm{AT}^{21}$ namely subjects are more likely to exceed AT in CLET compared to ILET. Therefore, the not-completed group who have a greater $\mathrm{V}_{\mathrm{CO} 2}$ production affected by reduced skeletal muscle could have exceeded AT and did not maintain exercise only in CLET.

\section{Limitations}

This study had several limitations. First, as the required sample size was not calculated, it was unclear whether the sample size had sufficient statistical power. The statistical power of this study for analyzing the difference in minimum VE/ $\mathrm{V}_{\mathrm{CO} 2}$ was calculated as $63.5 \%$ by post hoc analysis and it was slightly lower. Second, the changes in blood lactate and muscle strength of lower limbs, which may affect 
exercise tolerance and ventilation inefficiency, were not measured. Third, we did not evaluate training status or fitness level for modulating endurance exercise performance between two groups.

\section{Conclusion}

It was suggested that the respiratory and metabolic responses for determinants of endurance time in CLET were exercise beyond AT and increased $\mathrm{CO}_{2}$ output due to ventilatory inefficiency related to a reduced skeletal muscle in healthy adults. Therefore, those respiratory and metabolic responses of endurance time in CLET should be considered when we perform CLET. We will investigate whether the same results as in the present study will be shown in patients with COPD in the future.

\section{Declarations}

\section{Ethics approval and consent to participate}

All subjects were given an adequate explanation of the study and provided their written informed consent to participation. This study was conducted in accordance with the ethical principles for medical research involving human subjects of the Declaration of Helsinki after obtaining approval from the Shinshu University of Medical Ethics Committee (approval number: 3705).

\section{Consent for publication}

Not applicable.

\section{Availability of data and materials}

The datasets used and/or analysed during the current study are available from the corresponding author on reasonable request.

\section{Competing interests}

The authors declare that they have no competing interests 


\section{Funding}

This research received no specific grants from any funding agency in the public, commercial, or not-forprofit sectors.

\section{Authors' contributions}

SK performed the all measurements and analysis in writing the manuscript. Corresponding authors (KF) read and revised the manuscript.

\section{Acknowledgments}

The authors wish to thank Koganei Co., Ltd. (Tokyo, Japan) for providing the measurement equipment and facilities for this study, and M. Oyamada, a student of Shinshu University School of Health Sciences, for help and support. The authors and our colleagues thank the healthy adult volunteers who participated in the present study for their effort and enthusiastic cooperation.

\section{References}

1. Ross RM. ATS/ACCP statement on cardiopulmonary exercise testing. Am J Respir Crit Care Med. 2003: 167: 212-277.

2. Palange P, Ward SA, Carlsen KH, Casaburi R, Gallagher CG, Gosselink OR, O'donnel DE, PuenteMaestu L, Schols AM, Singh S, Whipp BJ. Recommendations on the use of exercise testing in clinical practice. Eur Respir J. 2007: 29: 185-209.

3. Whipp BJ, Ward SA, Lamarra N, Davis JA, Wasserman K. Parameters of ventilatory and gas exchange dynamics during exercise. J Appl Physiol. 1982: 52: 1506-1513.

4. O'donnel DE., Lam MIU, Webb K A. Measurement of symptoms, lung hyperinflation, and endurance during exercise in chronic obstructive pulmonary disease. Am J Respir Crit Care Med.1998: 158: 1557-1565.

5. Arizono S, Taniguchi H, Nishiyama O, Kondoh Y, Kataoka K, Ogawa T, Watanabe F, Nishimura K, Senjyu $\mathrm{H}$, Tabira K. Improvements in quadriceps force and work efficiency are related to improvements in endurance capacity following pulmonary rehabilitation in COPD patients. Intern Med. 2011: 50: 2533-2539.

6. O'Donnell DE, Casaburi R, Frith P, Kirsten A, De Sousa D, Hamilton A, Xue W, Maltais F. Effects of combined tiotropium/olodaterol on inspiratory capacity and exercise endurance in COPD. Eur Respir J. 2017: 49: 1601348. 
7. Bye PT, Esau SA, Levy RD, Shiner RJ, Macklem PT, Martin JG, Pardy RL. Ventilatory muscle function during exercise in air and oxygen in patients with chronic air-flow limitation. Am Rev Respir Dis.1985: 132: 236-240.

8. Oga T, Nishimura K, Tsukino M, Sato S. Exercise responses during endurance testing at different intensities in patients with COPD. Respir Med. 2004: 98: 515-521.

9. Andrianopoulos V, Wagers SS, Groenen MT, Vanfleteren LE, Franssen FM, Smeenk, FW, Vogiatzis I, Wouters EFM, Sprui MA. Characteristics and determinants of endurance cycle ergometry and sixminute walk distance in patients with COPD. BMC Pulm Med. 2014: 14: 97.

10. Berton DC, Reis M, Siqueira ACB, Barroco AC, Takara LS, Bravo DM, Andreoni S, Neder JA. Effects of tiotropium and formoterol on dynamic hyperinflation and exercise endurance in COPD. Respir Med. 2010: 104: 1288-1296.

11. Yamamoto J, Harada T, Okada A, Maemura Y, Yamamoto M, Tabira K. Difference in physiological components of VO2 max during incremental and constant exercise protocols for the cardiopulmonary exercise test. J phys ther sci. 2014: 26: 1283-1286.

12. Sasaki E, Nakamura M, Kida K, et al. Reference values for spirogram and blood gas analysis in Japanese non-smoking healthy adults. J Jpn Respir Soc. 2001: 39: 383-399.

13. Yamamoto M. Development of ramp protocol for use oftreadmill and a formula for prediction of oxygen uptake. Jpn J Appl Physiol. 1993: 23: 1 - 13 [in Japanese with English Abstract].

14. Ellestad M H, Thomas GS, Wann LS. Ellestad's Stress Testing: Principles and Practice. Oxford University Press, USA. 2018: 6: 440-441.

15. Dickstein K, Barvik S, Aarsland T, Snapinn S, Millerhagen J. Validation of a computerized technique for detection of the gas exchange anaerobic threshold in cardiac disease. Am J Cardiol. 1990: 66: 1363-1367.

16. Ghosh AK. Anaerobic threshold: its concept and role in endurance sport. Malays J Med Sci. 2004: 11: 24.

17. Wasserman K. The anaerobic threshold measurement to evaluate exercise performance. Am Rev Respir Dis. 1984: 129: 35-S40.

18. Nakade T, Adachi H, Murata M, Oshima S. Characteristics of patients with a relatively greater minimum VE/ $\mathrm{VCO}_{2}$ against peak $\mathrm{VO}_{2} \%$ and impaired exercise tolerance. Eur $\mathrm{J}$ Appl Physiol. 2018: 118:1547-1553.

19. Gonzales JU, Tucker SH, Kalasky MJ, Proctor DN. Leg strength is associated with ventilatory efficiency in older women. Int J Sports Med. 2012: 33: 537-542.

20. Keller-Ross ML, Chantigian DP, Evanoff N, Bantle AE, Dengel DR, Chow LS. VE/VCO2 slope in lean and overweight women and its relationship to lean leg mass. Int J Cardiol Heart Vasc. 2018: 21: 107110.

21. Ferri,A, Adamo S, La Torre A, Marzorati M, Bishop DJ, Miserocchi G. Determinants of performance in 1,500-m runners. Eur J Appl Physiol. 2012: 112: 3033-3043. 


\section{Tables}

Table 1. Subject characteristics of basic physical findings, lung function

\begin{tabular}{lcc} 
& Completed & Not-Completed \\
\hline$n$ & 17 & 10 \\
Age, years & $30.8 \pm 3.0$ & $26.9 \pm 3.3$ \\
Sex (male/female) & $15 / 2$ & $9 / 1$ \\
BMI, kg/m ${ }^{2}$ & $23.1 \pm 0.8$ & $21.1 \pm 0.9$ \\
$\%$ VC, $\%$ & $91.2 \pm 2.5$ & $91.9 \pm 4.9$ \\
$\% \mathrm{FEV}, \%$ & $98.9 \pm 2.4$ & $91.0 \pm 4.9$ \\
FEV $_{1} \angle \mathrm{FVC}, \%$ & $86.7 \pm 2.2$ & $87.7 \pm 2.4$
\end{tabular}

Notes: Values represent the means \pm standard error of the mean. The completed group is who continued to exercise for 20 minutes in constant load exercise test, and the not-completion group is who could not that.

Abbreviations: BMI, body mass index; VC, vital capacity; $\mathrm{FEV}_{1}$, forced expiratory volume in 1 second; FVC, forced vital capacity.

Table 2. The comararison of metabolic indexes in incremental load exercise test

\section{Completed Not-Completed}

$N$

$\mathrm{Vo}_{2}, \mathrm{ml} / \mathrm{kg} / \mathrm{min}$

$\mathrm{VCO}_{2}, \mathrm{ml} / \mathrm{kg} / \mathrm{min}$

RER

$\mathrm{ETo}_{2}, \%$

$\mathrm{ETcO}_{2}, \%$

$\mathrm{VE} / \mathrm{Vo}_{2}$

$\mathrm{VE} / \mathrm{VCO}_{2}$

VE, L/min

VT, L

$\mathrm{VD} / \mathrm{VT}$

$\mathrm{RR}$, breaths/min

$\mathrm{PR}$, beats/min
17

10
$54.2 \pm 2.5$
$60.1 \pm 4.0$

$1.25 \pm 0.02$

$1.28 \pm 0.02$

$14.1 \pm 0.6$

$15.2 \pm 0.8$

$5.7 \pm 0.6$

$5.4 \pm 0.6$

$37.8 \pm 0.9$

$39.1 \pm 1.5$

$$
30.2 \pm 0.6
$$

$30.6 \pm 1.1$

$107.6 \pm 5.3$

$113.2 \pm 6.7$

$2.3 \pm 0.1$

$2.5 \pm 0.2$

$$
0.26 \pm 0.01
$$

$0.25 \pm 0.02$

$47.2 \pm 1.4$

$45.9 \pm 2.3$

$148.1 \pm 40.2$

$151.5 \pm 37.2$ 
$\mathrm{Spo}_{2}, \%$

AT point, $\mathrm{ml} / \mathrm{kg} / \mathrm{min}$

RC point, $\mathrm{ml} / \mathrm{kg} / \mathrm{min}$

$\underline{\text { Minimum } \mathrm{VE} / \mathrm{VCO}_{2}}$

$$
91.2 \pm 4.0
$$

$$
26.0 \pm 1.1
$$$$
37.2 \pm 1.6
$$

$41.2 \pm 2.6$

Notes: All values of metabolic indexes except for AT point, RC point and minimum VE/ $\mathrm{Vco}_{2}$ are at peak of exercise. Values represent the means \pm standard error of the mean. The completed group who continued to exercise for 20 minutes in constant load exercise test and the non-completion group who could not that.

Abbreviations: $\mathrm{Vo}_{2}$, oxygen uptake; $\mathrm{VCO}_{2}$, carbon dioxide output; RER, respiratory exchange ratio; $\mathrm{ETo}_{2}$, end-tidal oxygen, ETco ${ }_{2}$, end-tidal carbon dioxide; VE, ventilation; VT, tidal volume; VD, dead-space gas volume; RR, respiratory rate; PR, pulse rate; $\mathrm{Spo}_{2}$, percutaneous oxygen saturation; $\mathrm{AT}$, anaerobic threshold; RC, respiratory compensation.

Table 3. The comararison of metabolic indexes in constant load exercise test

Completed

17

$19.5 \pm 0.1$

$35.2 \pm 1.8$

$34.3 \pm 1.9$

$0.97 \pm 0.01$

$14.1 \pm 0.6$

$5.7 \pm 0.6$

$33.3 \pm 5.0$

$34.6 \pm 4.1$

$35.9 \pm 4.9$

$\mathrm{VE} / \mathrm{VCO}_{2}$

VE, L/min

VT, L

VD/VT

RR, breaths/min

$\mathrm{PR}$, beats/min

$\mathrm{SpO}_{2}, \%$

$\underline{\text { Minimum } \mathrm{VE} / \mathrm{VCO}_{2}}$
Not-Completed

10

$9.9 \pm 2.0$

$41.5 \pm 2.6$

$42.7 \pm 3.1^{*}$

$1.01 \pm 0.02 *$

$15.2 \pm 0.8$

$5.4 \pm 0.6$

$$
76.4 \pm 4.1 \quad 92.2 \pm 7.0^{*}
$$

$1.76 \pm 0.08$

$2.19 \pm 0.20$

$0.27 \pm 0.02$

$0.26 \pm 0.03$

$43.9 \pm 1.9$

$43.7 \pm 3.8$

$159.8 \pm 6.3$

$177.4 \pm 4.2$

$91.2 \pm 1.0$

$90.7 \pm 2.3$

$\underline{27.0 \pm 2.9}$

$29.5 \pm 2.6 *$ 
completion group who could not that.

Abbreviations: $\mathrm{Vo}_{2}$, oxygen uptake; $\mathrm{Vco}_{2}$, carbon dioxide output; RER, respiratory exchange ratio; $\mathrm{ETo}_{2}$, end-tidal oxygen, ETco ${ }_{2}$, end-tidal carbon dioxide; VE , ventilation; VT, tidal volume;VD, dead-space gas volume; RR, respiratory rate; PR, pulse rate; $\mathrm{SpO}_{2}$, percutaneous oxygen saturation; AT, anaerobic threshold; RC, respiratory compensation.

Table 4. Single logistic regression analysis to predict completed exercise in constant load exercise test.

\begin{tabular}{llcc} 
Vriable & Odds ratio & $95 \% \mathrm{CI}$ & P-value \\
Minimum VE/ $/ \mathrm{VCO}_{2}$ & 0.707 & $0.50-0.10$ & 0.05 \\
RER & $0.001<0$ & $0-2.52$ & 0.64 \\
VE & 0.96 & $0.92-1.00$ & 0.06 \\
\hline
\end{tabular}

Note: Independent variable was completed or not-completed group as categorical variable.

Abbreviations: 95\% CI, 95\% confidence interval; $\mathrm{VE} / \mathrm{Vco}_{2}$, minute ventilation/carbon dioxide output; VE , minute ventilation.

\section{Figures}




\section{A)}

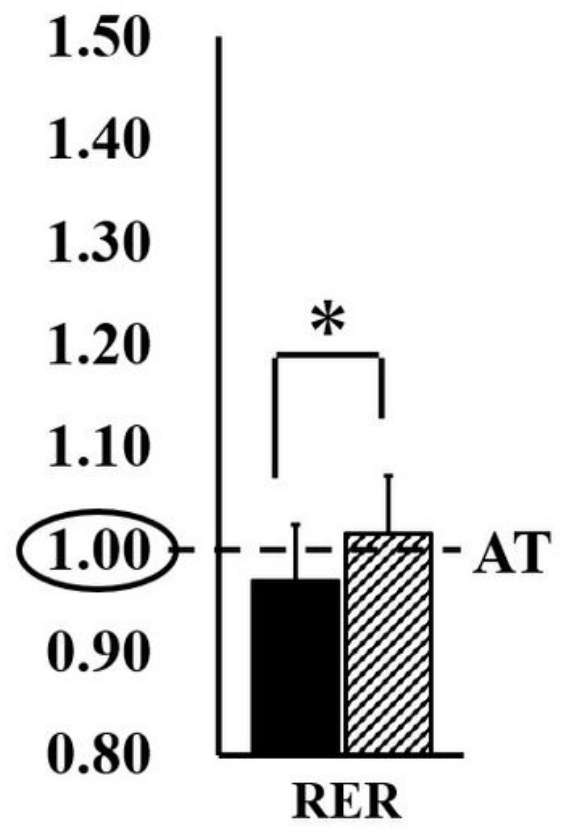

B)

(ml/ml) Completed group

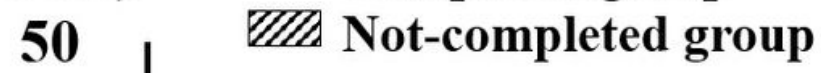

30

20

10

0

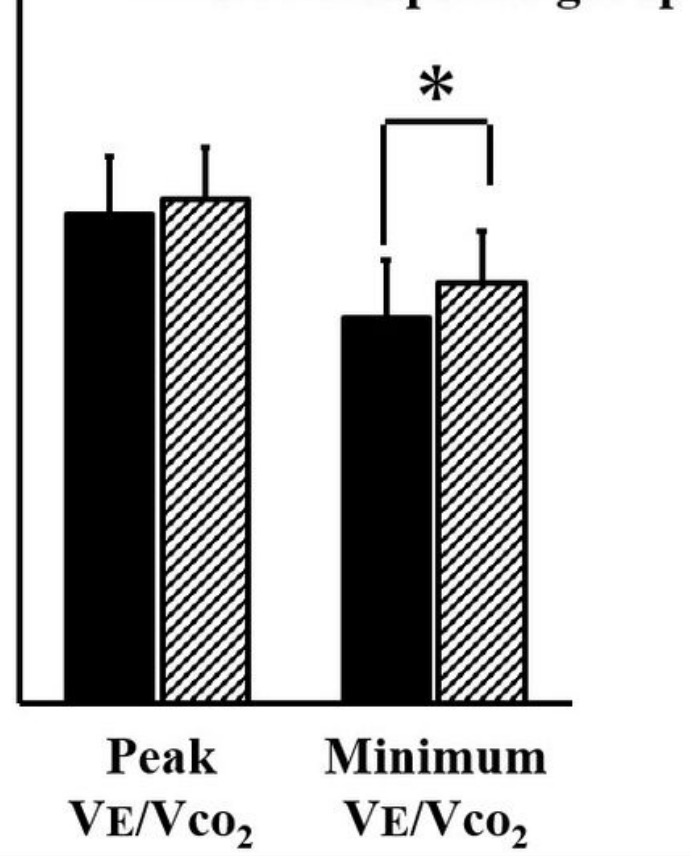

Figure 1

The comparison of gas exchange ratio and ventilation inefficiency indexes in constant load exercise test (CLET). Note: Panel A (left) shows the comparison of RER (Vco2/Vo2) as an index of AT in CLET. Panel B (right) shows the comparison of minimum VE/Vco2 as an index of ventilatory inefficiency in CLET. Abbreviations: AT, anerobic threshold; RER, respiratory exchange ratio; VE/Vco2, minute ventilation/carbon dioxide production. 